\title{
The impact of hyperbaric oxygen therapy on late radiation toxicity and quality of life in breast cancer patients
}

\author{
Marilot C. T. Batenburg ${ }^{1}$ (D) Wies Maarse ${ }^{2} \cdot$ Femke van der Leij $^{1} \cdot$ Inge O. Baas $^{3} \cdot$ Onno Boonstra $^{4} \cdot$ Nina Lansdorp $^{5}$. \\ Annemiek Doeksen ${ }^{6}$. Desiree H. J. G. van den Bongard ${ }^{7} \cdot$ Helena M. Verkooijen $^{8}$
}

Received: 21 December 2020 / Accepted: 12 July 2021 / Published online: 19 July 2021

(c) The Author(s) 2021

\begin{abstract}
Purpose To evaluate symptoms of late radiation toxicity, side effects, and quality of life in breast cancer patients treated with hyperbaric oxygen therapy (HBOT).

Methods For this cohort study breast cancer patients treated with HBOT in 5 Dutch facilities were eligible for inclusion. Breast cancer patients with late radiation toxicity treated with $\geq 20$ HBOT sessions from 2015 to 2019 were included. Breast and arm symptoms, pain, and quality of life were assessed by means of the EORTC QLQ-C30 and -BR23 before, immediately after, and 3 months after HBOT on a scale of 0-100. Determinants associated with persistent breast pain after HBOT were assessed.

Results $1005 / 1280$ patients were included for analysis. Pain scores decreased significantly from 43.4 before HBOT to 29.7 after 3 months $(p<0.001)$. Breast symptoms decreased significantly from 44.6 at baseline to 28.9 at 3 months follow-up $(p<0.001)$ and arm symptoms decreased significantly from 38.2 at baseline to 27.4 at 3 months follow-up $(p<0.001)$. All quality of life domains improved at the end of HBOT and after 3 months follow-up in comparison to baseline scores. Most prevalent side effects of HBOT were myopia (any grade, $n=576,57.3 \%)$ and mild barotrauma $(n=179,17.8 \%)$. Moderate/ severe side effects were reported in $3.2 \%(n=32)$ of the patients. Active smoking during HBOT and shorter time (i.e., median 17.5 vs. 22.0 months) since radiotherapy were associated with persistent breast pain after HBOT.

Conclusion Breast cancer patients with late radiation toxicity reported reduced pain, breast and arm symptoms, and improved quality of life following treatment with HBOT.
\end{abstract}

Keywords Breast cancer · Radiation toxicity · Hyperbaric oxygen therapy · Quality of life

\section{Introduction}

Around $68 \%$ of all women with breast cancer undergo radiotherapy as part of their treatment [1]. Even though radiotherapy techniques have improved over time, it still may—in

Marilot C. T. Batenburg

m.c.t.batenburg-3@umcutrecht.nl

1 Department of Radiation Oncology, University Medical Center Utrecht, Heidelberglaan 100, 3584 CX Utrecht, The Netherlands

2 Department of Plastic, Reconstructive and Hand Surgery, University Medical Center Utrecht, Utrecht, The Netherlands

3 Department of Oncology, University Medical Centre Utrecht, Utrecht, The Netherlands

4 Medical Director, Institute for Hyperbaric Oxygen Therapy, Rotterdam, The Netherlands combination with systemic therapy and surgery-induce late radiation toxicity [2-4]. Late radiation toxicity is characterized by a combination of breast or chest wall pain, breast and/or arm edema, fibrosis, impaired arm movement, telangiectasia, and impaired cosmetic outcome after radiotherapy.

5 DaVinci Kliniek for Hyperbaric Oxygen Therapy, Rotterdam, The Netherlands

6 Department of Surgery, St. Antonius Ziekenhuis, Nieuwegein, The Netherlands

7 Department of Radiation Oncology, Amsterdam University Medical Centers, Amsterdam, The Netherlands

8 Imaging Division, University Medical Center Utrecht, Utrecht University, Utrecht, The Netherlands 
Symptoms such as fibrosis and breast pain may continue to increase during at least 10 years after radiotherapy and substantially impair daily functioning and quality of life [5].

Treatment of late radiation toxicity depends on the symptoms and may consist of analgesics, physiotherapy, lymphedema therapy, and in some cases (reconstructive) surgery. Another proposed treatment for late radiation toxicity is hyperbaric oxygen therapy (HBOT). During HBOT, patients inhale $100 \%$ oxygen in a hyperbaric chamber with increased air pressure. The combination of oxygen and increased air pressure induces neovascularization and stimulates formation of collagen by fibroblasts [6, 7]. HBOT has been proven a safe and effective treatment for late radiation toxicity in different tumor sites [8-10]. For that reason, HBOT for late radiation toxicity is endorsed by insurers in the Netherlands. However, evidence for the effectivity of HBOT in breast cancer patients with late radiation toxicity is limited [11, 12]. Consequently, in the Netherlands, HBOT is mostly used as a treatment option for late radiation toxicity in breast cancer patients who insufficiently benefited from analgesics, physiotherapy, or lymphedema therapy.

The aim of this cohort study was to evaluate patientreported late radiation toxicity in breast cancer patients treated with HBOT between 2015 and 2019 in one center providing hyperbaric oxygen therapy in the Netherlands. Secondly, side effects after HBOT, quality of life, and factors associated with effectivity of treatment were assessed.

\section{Methods}

All breast cancer patients with late radiation toxicity referred between January 2015 and December 2019 for HBOT in the Institute for Hyperbaric Oxygen Therapy (IvHG) were eligible for inclusion. The IvHG has five locations in the Netherlands. Patients who provided written consent for the use of their data for research purposes were included. Patients referred to the IvHG who were found to be ineligible for HBOT (e.g., due to comorbidities), patients treated with $<20$ HBOT sessions, or patients referred for re-treatment with HBOT were excluded. Also, patients with osteoradionecrosis and patients treated with HBOT prior to surgery were excluded, as they were treated with a different number of HBOT treatment sessions. Prior to HBOT, a physician confirmed late radiation toxicity and determined if the breast or chest wall symptoms (i.e., a combination of breast or chest wall pain, breast and/or arm edema, fibrosis, impaired arm movement, telangiectasia, and impaired cosmetic outcome) were likely to be the result of radiotherapy. After data collection, the complete dataset was anonymized and transferred to the division of Imaging and Oncology of the UMCU to ensure independent analysis. Data analysis was performed by independent researchers of the UMCU. Staff of the IvHG had no role in study design or decision to file the manuscript for publication. The institutional review board of the University Medical Center Utrecht (UMCU) approved this study.

\section{Hyperbaric oxygen therapy}

Standard HBOT consisted of 40 treatment sessions (1 session/day, 5 days/week) at 2.5 atmospheres absolute (ATA), with a duration of $115 \mathrm{~min}$ per session (10 min compression, 4 times 20 min $100 \%$ oxygen with breaks of 5 min, and 10 min decompression) [13]. HBOT is administered in a high-pressure chamber. After reaching the desired treatment pressure (2.5 ATA), the patient starts breathing $100 \%$ oxygen by a closed built-in breathing system (either a hood or a mask). For safety reasons, the chamber is only filled with air under pressure and the patient always breathes oxygen by a closed system. Patients may receive more or less treatment sessions. For example, treatment effect is evaluated with the HBO physician after 30 treatment sessions. If no treatment effect was seen after 30 sessions, patients could stop HBO treatment after 30 sessions. Also, patients may receive more or less than $40 \mathrm{HBO}$ sessions for other reasons related to $\mathrm{HBO}$ (i.e., side effects, sufficient results prior to 40 sessions) or not related to HBO (i.e., planned vacation, medical problems not related to $\mathrm{HBO}$, personal circumstances). Therefore, reasons for treatment sessions other than 40 were recorded. At 3 months after the last HBO session, patients were contacted by phone and received the European Organization for Research and Treatment of Cancer Quality of Life Questionnaire (EORTC QLQ).

\section{Data collection}

Patient, treatment, and tumor characteristics, HBO treatment details, and side effects were extracted from the individual patient records. Patient-reported outcome measures were collected as part of routine clinical care. All data were entered into a database by a research nurse. In accordance with a data collection protocol designed by the UMCU research team, data from the patient files were entered into a standardized case report form. Quality of data extraction was regularly monitored by comparing CRFs with the source documents (around 32 cases, 3\%).

\section{Outcome measurements}

\section{Patient-reported outcome measurements}

Breast/chest wall and arm symptoms, pain, and quality of life were collected as part of standard care using the EORTC QLQ. The EORTC QLQ comprises 30 quality of life and functioning items (C30) as well as 23 breast-specific items (BR23) [14]. All items were scored on a 4-point Likert scale. 
Total scores (0-100) for subscales of the EORTC questionnaires were calculated using the EORTC scoring manual. For functional scales, a higher score indicated a better outcome. For symptom scales, a higher score indicated more symptoms. Breast symptoms were evaluated using four questions on pain, swelling, sensitivity, and skin problems in the affected breast or chest wall (BR23). The arm symptom scale is based on 3 items: pain and swelling in arm or shoulder and difficulty to move the arm up or sideways. The EORTC QLQ questionnaires were used as part of standard treatment. Patients received questionnaires at predefined time-points, i.e., prior to treatment (baseline), after the last $\mathrm{HBO}$ session ( 2 months after baseline), and at 3 months after the last $\mathrm{HBO}$ session ( 5 months after baseline).

\section{Cohort outcomes and side effects}

Side effects of HBOT were evaluated by the HBO physician during follow-up visits (i.e., after 15, 30, and 40 sessions and by telephone at 3 months after the end of HBOT). Side effects after HBOT may include barotrauma, hypoglycemia, myopia, fatigue, cataract, sinus squeeze, (acute or chronic) oxygen toxicity, cardiac decompensation/heart failure, decompression disease, or pneumothorax. Otoscopy was only performed in case of ear pain or repetitive trouble in equalizing middle ear pressure. Then, barotrauma was classified according to the 6-point MacFie classification (also known as modified TEED classification): no abnormalities with otoscopy (grade 0 ), increased vessel visibility around the eardrum (without/with minor/with major bleeding, grade 1-3), blood in middle ear (grade 4), or eardrum perforation (grade 5) [15-17]. All side effects were standardly evaluated during visits with the HBOT physician. However, no grading system was available for other side effects than barotrauma. For this study, fatigue was evaluated using the EORTC QLQ-C30 fatigue subscale. A fatigue score $\geq 71$ was considered clinically relevant, based on the Thresholds for Clinical Importance of Giesinger et al. [18]. Newly developed (clinically relevant) fatigue during HBOT or at follow-up was considered to be a side effect of the HBOT. Barotrauma grade 0-2, hypoglycemia, myopia, and fatigue were classified as mild side effects, as they are transient in nature [19]. Moderate or severe side effects were cataract, barotrauma grade 3-5, sinus squeeze, (acute or chronic) oxygen toxicity, cardiac decompensation/heart failure, decompression disease, or pneumothorax.

\section{Statistics}

Patient characteristics, breast cancer treatment, HBO treatment characteristics, and side effects were described using frequencies and proportions for categorical data and for continuous data means with standard deviation for normally distributed data and medians with interquartile ranges (IQR) were used for skewed data.

Paired T tests or Wilcoxon rank test-depending on distribution-were used to compare pain, breast symptoms, and arm symptoms between baseline (T0) and T1 (end treatment) and between T0 and T2 (follow-up), respectively. Analysis was performed using all available questionnaires. For sensitivity analysis, complete case analysis was performed. To evaluate the association between patient and treatment characteristics and persistence of breast pain after HBOT, the EORTC QLQ-BR23 item on breast pain was used (item 50, "Have you had any pain in the area of your affected breast?"). Breast pain was dichotomized into moderate/severe pain and no/mild pain. Patients with persistent moderate/severe breast pain after HBOT were categorized as unsuccessful therapy (no pain response). Descriptive statistics were used to evaluate characteristics associated with adequate treatment effect, i.e., mild or no pain at follow-up. Statistical Package for Social Sciences (SPSS) software version 25 was used for analysis. A $p$-value $<0.05$ was considered significant.

\section{Results}

Between January 2015 and December 2019, 1280 breast cancer patients were referred for HBOT. Of those, 1005 (78.5\%) patients were included for analysis (Fig. 1). The most common reasons for exclusion were ineligibility for HBOT $(n=114)$, treatment with $<20$ HBOT sessions $(n=61)$, and no consent for the use of data for research $(n=46)$. The response rate to the EORTC questionnaire was $95 \%$ at baseline, $85 \%$ at the end of treatment, and $58 \%$ after 3 months follow-up. The majority of patients were female ( $n=1002,99.7 \%$ ) (Table 1$)$. The mean age was 57.9 years and most patients were treated with breast-conserving surgery $(n=731,73 \%)$. The most common radiotherapy fractionation schedule was 15-19 fractions without boost $(n=231,23.0 \%)$ or $21-24$ fractions with boost $(n=176$, $17.5 \%)$. In total, $336(33.4 \%)$ patients received local radiotherapy and $264(26.3 \%)$ patients received locoregional radiotherapy (i.e., radiation therapy on periclavicular and/ or axillary lymph nodes). During HBOT, $13 \%(n=134)$ of the patients were active smokers and $41 \%(n=413)$ were former smokers. The time since radiotherapy ranged from 1 to 582 months (median 22 months). Patients who responded to all questionnaires were, on average, older (mean age 59.0 vs. 56.8) and had a longer time since radiotherapy (median 48 months vs. 37 months) than non-responders (Supplementary material Table 1).

The number of HBO sessions ranged from 20 to 60 (median 40$) ; 73.1 \%(n=735)$ of the patients received 40 HBO sessions (Table 2). Reasons for undergoing less HBOT 
Fig. 1 Flowchart of patients included for analysis after inand ex-clusion criteria

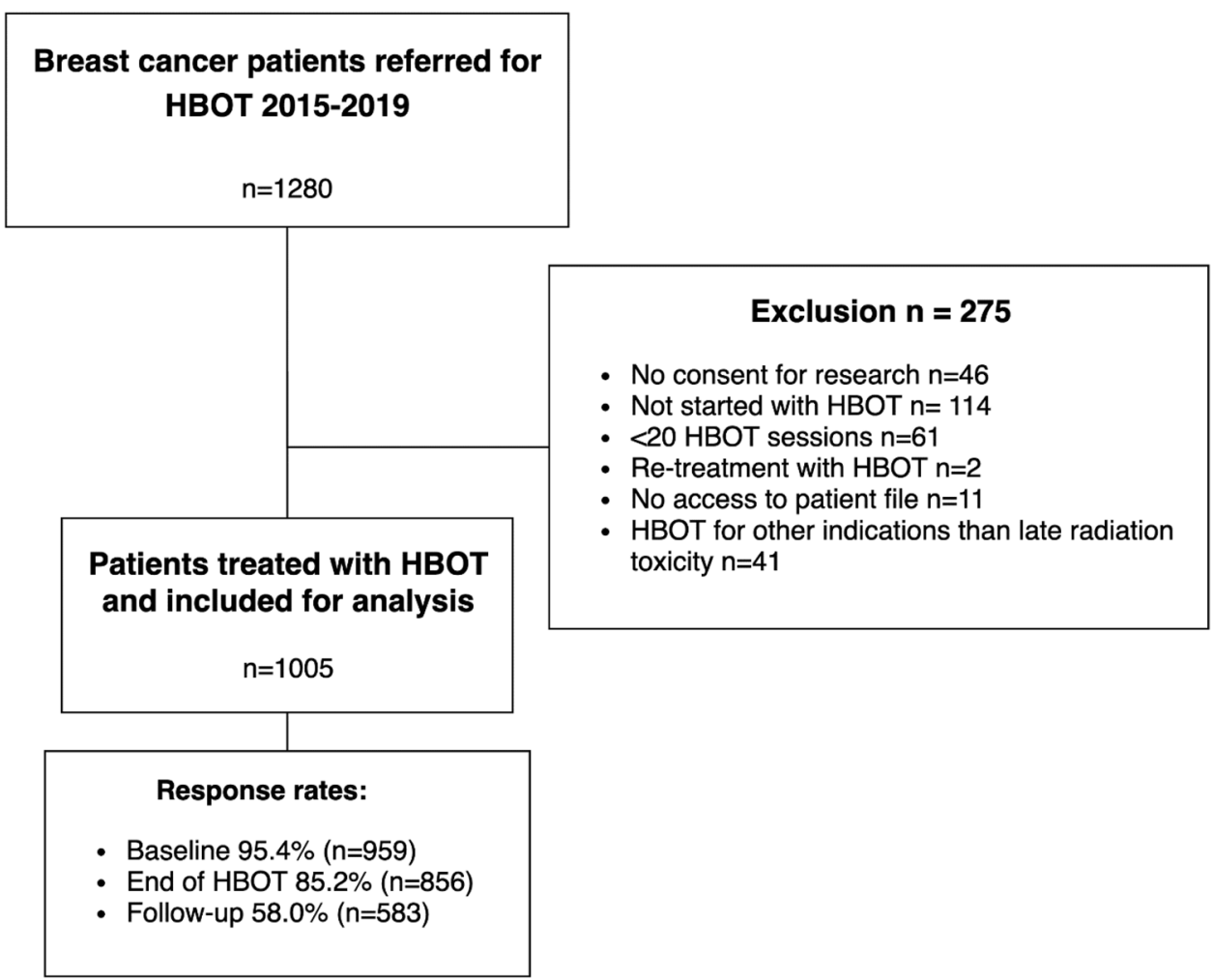

than planned were personal circumstances $(n=53)$, sufficient results $(n=31)$, or medical problems not related to HBOT $(n=29)$. There were 32 patients who stopped HBOT early due to no or insufficient results and 17 patients who stopped due to complications of HBOT. In total, 30 patients received $>40$ HBOT sessions, mostly due to disruption of treatment sessions $(n=13)$. The most common side effects of HBOT were (transient) myopia $(n=576,57 \%)$ and mild barotrauma ( $n=179,18 \%$ ) (Table 2$)$. Moderate/severe side effects were reported by 32 patients: oxygen toxicity $(n=4$, $0.4 \%)$, barotrauma grade $3-4(n=26,2.6 \%)$, sinus squeeze $(n=1,0.1 \%)$, and cataract $(n=1,0.1 \%)$.

Pain scores decreased significantly from 43.4 prior to HBOT to 30.5 at the end of HBOT $(p<0.001)$ to 29.7 at 3 months follow-up $(p<0.001)$ (Fig. 2). Also, a significant reduction in breast symptom scores at the end of HBOT (29.4) and 3 months follow-up (28.9) was seen in comparison to baseline score (44.6) $(p<0.001)$. Arm symptom scores reduced significantly $(p<0.001)$ from 38.2 to 26.0 at the end of treatment and 27.4 after 3 months follow-up. Repeating the analysis in the subgroup of 352 patients who completed questionnaires at all timepoints did not change the results (Supplementary table 2). Role functioning scores improved from 62.7 at baseline to 67.0 immediately after HBO and 73.2 after 3 months follow-up (Fig. 3). Social functioning scores improved from 74.2 prior to treatment to 75.9 after treatment and further to 82.3 after 3 months follow-up. Also, emotional functioning, physical functioning, and quality of life scores increased over time.

EORTC breast pain scores were available at baseline and at the end of HBOT for 749 patients. In total, $61.5 \%$ $(n=461 / 749)$ of the patients reported breast pain grade 3-4 prior to treatment and $30.0 \%(n=225 / 749)$ reported breast pain grade 3-4 after HBOT. Of the patients with pain grade $3-4$ at baseline, 271 patients $(58.8 \%)$ had grade $1-2$ pain at end of treatment and 190 patients still had pain grade 3-4 (i.e., treatment failures) after HBOT (Table 3). Factors associated with treatment success were smoking and time since radiotherapy. Of the patients who smoked during HBOT, $45 \%$ ( $n=29 / 64)$ had good response (i.e., no/mild pain after HBOT $), 61 \%(n=121 / 199)$ of the never smokers and $61 \%$ of the former smokers ( $n=120 / 198)$ had good response to HBOT. The median time since radiotherapy was 22 months in the group with good response to HBOT and 17.5 months in the group with persistent pain after HBOT.

\section{Discussion}

In this large cohort study of breast cancer patients with late radiation toxicity, a reduction of pain, breast and arm symptoms, and an improvement in patient-reported outcomes (i.e., quality of life and social, role, emotional, and physical functioning) following treatment with HBOT was seen. The majority of the patients in this study experienced some 
Table 1 Baseline characteristics

\begin{tabular}{|c|c|}
\hline & $n=1005$ \\
\hline Age [mean (SD)] & $57.9(9.7)$ \\
\hline Female gender & $1002(99.7)$ \\
\hline \multicolumn{2}{|l|}{ Pathological tumor stage $^{\mathrm{a}}$} \\
\hline 0 & $4(0.4)$ \\
\hline In situ & $34(3.4)$ \\
\hline 1 & $456(45.4)$ \\
\hline 2 & $246(24.5)$ \\
\hline 3 & $56(5.6)$ \\
\hline 4 & $18(1.8)$ \\
\hline Unknown & $191(19)$ \\
\hline \multicolumn{2}{|l|}{ Type of surgery } \\
\hline Breast-conserving surgery & $731(72.7)$ \\
\hline Mastectomy without breast reconstruction & $180(17.9)$ \\
\hline Autologous breast reconstruction & $36(3.6)$ \\
\hline Implant breast reconstruction & $29(2.9)$ \\
\hline Breast reconstruction, unknown type & $17(1.7)$ \\
\hline Unknown & $12(1.2)$ \\
\hline \multicolumn{2}{|l|}{ Axillary surgery ${ }^{\mathrm{a}}$} \\
\hline Axillary lymph node dissection & $257(25.5)$ \\
\hline Sentinel Node Procedure & $569(56.6)$ \\
\hline Other & $10(1.0)$ \\
\hline No axillary treatment/unknown & $169(16.8)$ \\
\hline \multicolumn{2}{|l|}{ Systemic treatment } \\
\hline Chemotherapy alone & $161(16.0)$ \\
\hline Hormonal therapy alone & $106(10.5)$ \\
\hline Both chemotherapy and hormonal therapy & $464(46.2)$ \\
\hline No adjuvant treatment & $241(24.0)$ \\
\hline Unknown & $33(3.3)$ \\
\hline \multicolumn{2}{|l|}{ Smoking } \\
\hline Never & $455(45.3)$ \\
\hline Current smoker & $134(13.3)$ \\
\hline Previous smoker & $413(41.1)$ \\
\hline Unknown & $3(0.3)$ \\
\hline \multicolumn{2}{|l|}{ Diabetes Mellitus } \\
\hline Yes & $83(8.3)$ \\
\hline No & $922(91.7)$ \\
\hline Body Mass Index (median (IQR)) ${ }^{\mathrm{b}}$ & $27.4(7.1)$ \\
\hline Unknown & $228(25.3)$ \\
\hline \multicolumn{2}{|l|}{ Type of radiation therapy } \\
\hline Local & $336(33.4)$ \\
\hline Locoregional & $264(26.3)$ \\
\hline Unknown & $405(40.3)$ \\
\hline \multicolumn{2}{|l|}{ Radiotherapy boost ${ }^{\mathrm{c}}$} \\
\hline Yes & $372(39.4)$ \\
\hline No & $396(37.0)$ \\
\hline Unknown & $237(23.6)$ \\
\hline \multicolumn{2}{|l|}{ Radiotherapy fractionation $^{\mathrm{d}}$} \\
\hline $6-12$ fractions & $15(1.5)$ \\
\hline $15-19$ fractions & $231(23.0)$ \\
\hline 21-24 fractions, with boost & $176(17.5)$ \\
\hline
\end{tabular}

Table 1 (continued)

\begin{tabular}{ll}
\hline & $n=1005$ \\
\hline $20-25$ fractions, no boost & $122(12.1)$ \\
$>26$ fractions & $88(8.8)$ \\
Unknown & $373(37.1)$ \\
Previous radiotherapy breast/chest wall ${ }^{\mathrm{a}}$ & \\
Yes & $51(5.1)$ \\
No & $699(69.6)$ \\
Unknown & $255(25.4)$ \\
Months since radiotherapy [median (IQR)] & $22(35)$ \\
\hline
\end{tabular}

Numbers are shown as $\mathrm{n}(\%)$ unless stated otherwise. Continuous outcomes are shown as mean (SD) when normally distributed and median(IQR) otherwise

$S D$ standard deviation, IQR interquartile range

${ }^{\text {a }}$ Total other than $100 \%$ due to rounding

${ }^{\mathrm{b}}$ Calculated as weight/height ${ }^{2}$

${ }^{\mathrm{c}}$ An additional radiotherapy boost on the tumor bed or axillary/lymph node boost

${ }^{\mathrm{d}}$ Dose per fraction was unknown

side effects of HBOT. The most common side effects were (transient) myopia and mild barotrauma. Myopia and mild barotrauma are transient side effects and disappear mostly in the first three months after HBOT. This study confirmed that HBOT is a safe treatment, as severe side effects were seen in $3.6 \%$ of all patients and mostly concerned barotrauma's.

Two previous studies evaluated the effect of HBOT for breast cancer patients with late radiation toxicity. In the prospective cohort study by Carl et al., outcomes of 32 breast cancer patients treated with HBOT were compared with 12 control patients who refused HBOT [12]. Late radiation toxicity was evaluated using the LENT-SOMA scores on a 4-point Likert scale. Similar to our study, a significant reduction in pain was seen after HBOT. Eleven months after treatment, median pain scores for the HBOT group decreased from 3 (range 1-4) prior to HBOT to 0 (range $0-2$ ). The median pain score in the observational group remained stable at grade 3 over time. Like us, Carl et al. reported a significant reduction of edema after HBOT. This reduction of edema was not seen in the control group. In contrast to our study, no effect on physician-reported fibrosis was reported by Carl. et al. In the study by Carl et al., the median fibrosis score was already 0 in both groups prior to the study; so, no effect of HBOT on fibrosis could be seen.

In the prospective study by Teguh et al., 57 patients with late radiation toxicity received on average $47 \mathrm{HBO}$ sessions on 2.4 ATA [11]. Late radiation toxicity was evaluated by means of the EORTC QLQ-C30 and -BR23. Moderate/ severe breast pain was seen in $66.7 \%$ of the patients prior to HBOT, which is similar to $61.5 \%$ in our study. At the end of HBOT, $14.5 \%$ of the patients reported moderate/severe pain. This proportion was $30.0 \%$ in our study. In the study from 
Table 2 Number of hyperbaric oxygen treatment sessions, reasons for treatment sessions $<40$, and side effect of hyperbaric oxygen therapy

\begin{tabular}{|c|c|}
\hline Number of HBO sessions & $n=1005$ \\
\hline HBO sessions [median(range)] & $40(20-60)$ \\
\hline$<40$ sessions $(\mathrm{n}(\%))$ & $240(23.9)$ \\
\hline 40 sessions $[\mathrm{n}(\%)]$ & $735(73.1)$ \\
\hline$>40$ sessions $[\mathrm{n}(\%)]$ & $30(3.0)$ \\
\hline Reasons for treatment sessions $<40$ & $n(\%)$ \\
\hline Sufficient results & $31(13)$ \\
\hline No/insufficient results & $32(13)$ \\
\hline Complications of HBOT & $17(7)$ \\
\hline Private circumstances & $53(22)$ \\
\hline Medical problems not related to HBOT & $29(12)$ \\
\hline Unclear & $78(33)$ \\
\hline Total & $240(100)$ \\
\hline \multicolumn{2}{|l|}{ Side effects of HBOT } \\
\hline Number of patients with side effects [n (\%)] & $697(69.4)$ \\
\hline Number of side effects & 882 \\
\hline Mild (transient) side effects & $n(\%)$ \\
\hline Barotrauma grade $0-2^{\mathrm{a}}$ & $179(17.8)$ \\
\hline Hypoglycemia & $2(0.2)$ \\
\hline Myopia & $576(57.3)$ \\
\hline Fatigue (newly developed) & $52(5.2)$ \\
\hline Complication, unclear & $41(4.1)$ \\
\hline Moderate/severe side effects & $n(\%)$ \\
\hline Cataract $^{\mathrm{b}}$ & $1(0.1)$ \\
\hline Barotrauma grade $3-4^{\mathrm{a}}$ & $26(2.6)$ \\
\hline Barotrauma sinus squeeze & $1(0.1)$ \\
\hline Oxygen toxicity & $4(0.4)$ \\
\hline
\end{tabular}

No cases: chronic oxygen toxicity, cardiac decompensation/heart failure, decompression disease, hypoxia, deceased, pneumothorax. Fatigue was calculated as number of patients with newly developed fatigue during HBOT [i.e., fatigue scores higher than 40 (18)]

HBOT hyperbaric oxygen therapy

${ }^{a}$ In accordance with the Macfie classification

${ }^{\mathrm{b}}$ Cataract may be therapy induced or pre-existent

Fig. 2 The effect of hyperbaric oxygen therapy on pain, breast symptoms, and arm symptoms. A higher score indicates more symptoms. *Significant difference $(p<0.05)$ tested with Wilcoxon rank test.

Time: $0=$ baseline (i.e., prior to HBOT), $2=$ end of HBOT, $5=3$ months after HBOT

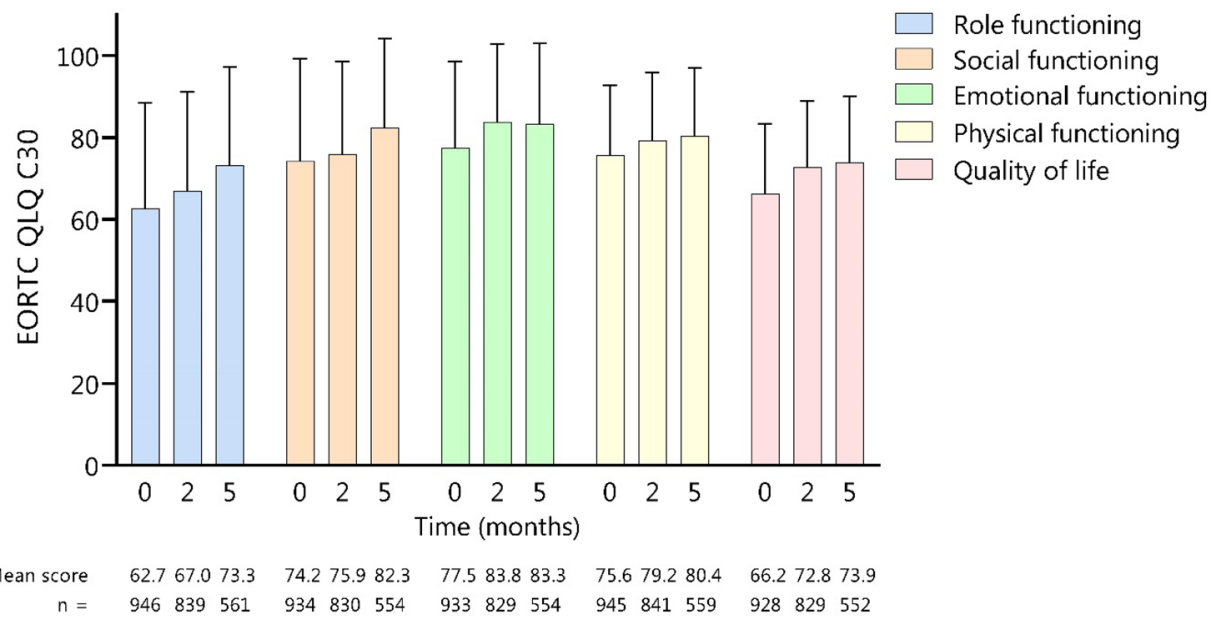

Time: 0 = baseline (i.e. prior to HBOT), 2 = end HBOT, 5 = three months after HBOT 
Fig. 3 The effect of hyperbaric oxygen therapy on quality of life scores and role, emotional, social, and physical functioning using the EORTC QLQ-C30 questionnaire. A higher score indicates a better quality of life. Time: $0=$ baseline (i.e., prior to HBOT), 2 = end of HBOT, $5=$ three months after HBOT

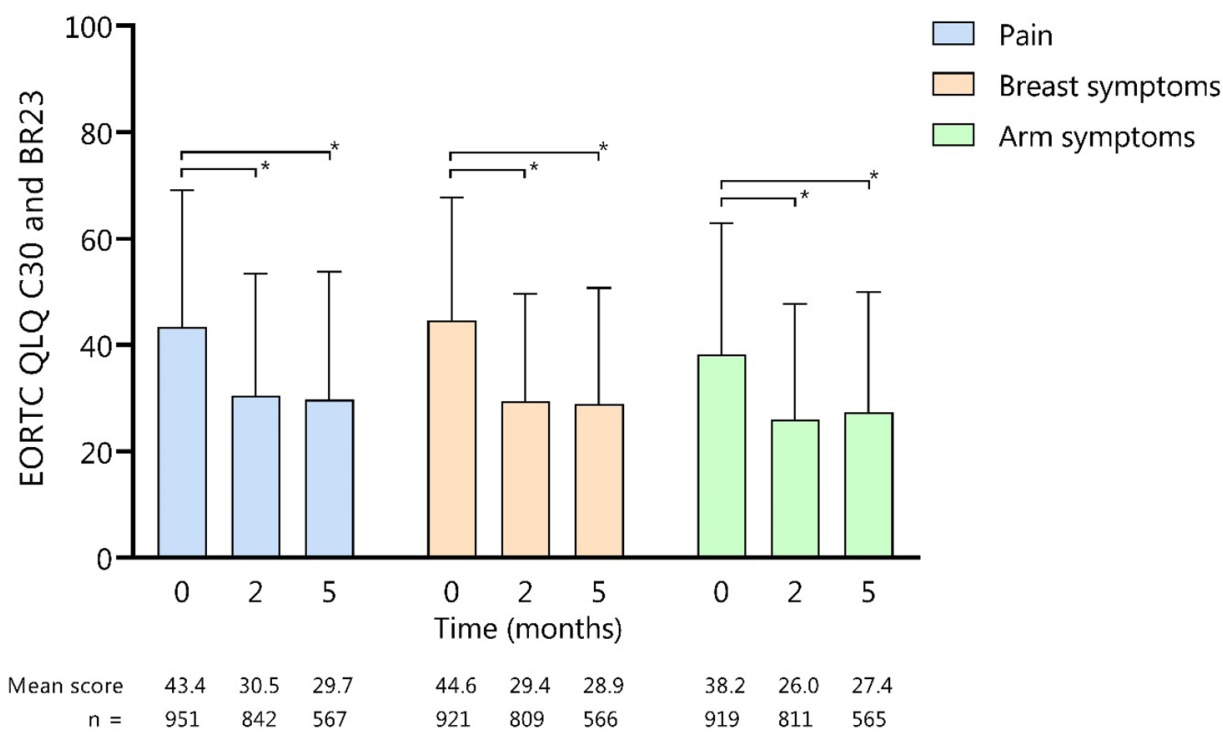

*Significant difference $(p<0.05)$ tested with Wilcoxon rank test

Time: 0 = baseline (i.e. prior to HBOT), 2 = end HBOT, $5=3$ months after HBOT
Teguh et al., $51 \%$ of the patients received chemotherapy and $6 / 57(11 \%)$ of the patients had no surgery in contrast to, respectively, $72 \%$ and at most $1.2 \%$ in our population. Consequently, there might be more fibrosis in our population and treatment with HBOT could therefore have been less effective. Proportions of moderate/severe swelling of breast and arm and problems with moving the arm prior to HBOT and after HBOT in the study of Teguh et al. were comparable to our study.

In our study, pain response was defined as a decrease in pain from grade 3-4 to 1-2 after HBOT. The proportion of patients that still experienced pain after HBOT was higher in the group of patients that actively smoked in comparison to patients who were never or former smokers. HBOT induces neo-vascularization and smoking might damage these newly developed vessels [6]. Consequently, patients who actively smoke during treatment might have less effect of the treatment and experience persisting breast pain after HBOT. In addition, the interval between radiotherapy and HBOT was slightly larger (i.e., difference of 5 months) for patients with breast pain response than for patients with persistent pain after HBOT. A possible explanation is that when radiotherapy is longer ago, it could be more straightforward to differentiate late radiation toxicity from side effects of other breast cancer treatments. As HBOT is specifically targeted for late radiation toxicity, better selection of patients eligible for HBOT may lead to better treatment results. Also, patients who suffered longer from breast pain may report a larger difference in breast pain as they are more relieved than patients who suffered breast pain shortly.

Our study suffers from several limitations: first, clinical outcome data were collected retrospectively, which may have introduced some room for information bias. For example, there may be an underestimation of side effects of HBOT as, theoretically, not all physicians consequently reported side effects in the patient records. To ensure data quality, independent monitoring of extracted data was performed. While monitoring, no discrepancies in extracted data and source date were seen. Second, despite a very high response rate at baseline and at the end of treatment, the response rate at 3 months after the end of treatment was suboptimal $(58 \%)$. This is partly due to the fact that not all patients were contacted at 3 months after HBOT. Also, the response rate depends on the response of the patients to the EORTC QLQ. In case the response was selective, this may have overor under-estimated the impact of HBOT on PROs. Some patient characteristics differed between non-responders and responders, as non-responders were on average older and received radiotherapy longer ago. Also, the reason for nonresponse is unknown. Therefore, the effect of HBOT could have been different for non-responders than responders. Third, no long-term follow-up was available for this study and no control group was included. Potentially, symptoms and quality of life could also have improved over time (i.e., regressed to the mean) without treatment of HBOT [20, 21]. As there was no control group, no distinction could be made between regression to the mean and the effect of HBOT. Therefore, the study results need to be confirmed in a randomized controlled trial in order to compare HBOT to a control group. For that reason, we are currently conducting a randomized controlled trial following the Trials within Cohorts design in our institute (NCT04193722) [22]. In this trial, the effect of HBOT on late radiation toxicity is compared to usual care in breast cancer patients. 
Table 3 Characteristics of patients with and without persistent breast or chest wall pain after hyperbaric oxygen therapy

\begin{tabular}{|c|c|c|}
\hline & Pain response $(n=271)$ & $\begin{array}{l}\text { No pain } \\
\text { response } \\
(n=190)\end{array}$ \\
\hline Age [mean (SD)] & $57.9(9.7)$ & $57.4(8.9)$ \\
\hline \multicolumn{3}{|l|}{ Type of surgery } \\
\hline Breast-conserving surgery & $206(57)$ & $153(43)$ \\
\hline Mastectomy without breast reconstruction & $49(66)$ & $25(34)$ \\
\hline Mastectomy followed by breast reconstruction ${ }^{a}$ & $13(57)$ & $10(44)$ \\
\hline Unknown & $3(60)$ & $2(40)$ \\
\hline \multicolumn{3}{|l|}{ Systemic treatment } \\
\hline Chemotherapy alone & $40(56)$ & $31(44)$ \\
\hline Hormonal therapy alone & $38(62)$ & $23(38)$ \\
\hline Both chemotherapy and hormonal therapy & $115(60)$ & $76(40)$ \\
\hline No (neo)adjuvant treatment & $68(54)$ & $58(46)$ \\
\hline \multicolumn{3}{|l|}{ Smoking } \\
\hline Never & $121(61)$ & $77(39)$ \\
\hline Current smoker & $29(45)$ & $35(54)$ \\
\hline Previous smoker & $120(61)$ & $78(39)$ \\
\hline Unknown & $1(100)$ & $0(0)$ \\
\hline \multicolumn{3}{|l|}{ Diabetes Mellitus } \\
\hline Yes & $18(55)$ & $15(46)$ \\
\hline No & $253(59)$ & $175(41)$ \\
\hline Body Mass Index (median IQR) ${ }^{\mathrm{b}}$ & $27.9(7.1)$ & $26.6(7.2)$ \\
\hline \multicolumn{3}{|l|}{ Radiotherapy boost } \\
\hline Yes & $98(57)$ & $75(43)$ \\
\hline No & $111(63)$ & $64(37)$ \\
\hline Unknown & $62(55)$ & $51(45)$ \\
\hline Months since radiotherapy [median(IQR)] & $22(34)$ & $17.5(30)$ \\
\hline
\end{tabular}

Numbers are shown as $\mathrm{n}(\%)$ unless stated otherwise. Continuous outcomes are shown as mean (SD) when normally distributed and median (IQR) otherwise

Patients with breast pain grade 3-4 (EORTC QLQ 50) at baseline were selected. Patients without breast pain were defined as breast pain grade $1-2$ at end of HBOT. Patients with breast pain were defined as patients with grade $3-4$ breast pain at the end of HBOT

$S D$ standard deviation, IQR interquartile range

${ }^{\text {a }}$ Total other than $100 \%$ due to rounding

${ }^{\mathrm{b}}$ Calculated as weight $/$ height $^{2}$
In conclusion, this large study of consecutive breast cancer patients with late radiation toxicity shows a beneficial effect of HBOT on patient-reported symptoms and quality of life and functioning until at least three months after HBOT. Also, it confirms that hyperbaric oxygen therapy is safe, as severe side effects were limited. The most common side effects were (reversible) myopia and mild barotrauma. Due to the non-comparative design of the study, these results need to be confirmed in a randomized controlled trial.

Supplementary Information The online version contains supplementary material available at https://doi.org/10.1007/s10549-021-06332-2.
Acknowledgements The authors thank Denise Joseph for her contribution to the data collection.

Funding This project was funded by a grant from Vrienden van het UMC Utrecht. Vrienden van het UMC Utrecht had no role in the design of this study, data collection, analysis or interpretation of the data, or decision to file this manuscript for publication.

Data availability Data are available upon request.

Code availability Data were analyzed anonymously. Code was available by the research nurse of the IvHG. 


\section{Declarations}

Conflict of interest N. Lansdorp is hyperbaric physician and O. Boonstra is medical director of the IvHG.

Ethical approval The Medical Ethics Committee of the University Medical Center Utrecht approved this study (reference number 20-183).

Consent to participate Informed consent was obtained from all participants.

Consent for publication All patients signed informed consent regarding publishing their data.

Open Access This article is licensed under a Creative Commons Attribution 4.0 International License, which permits use, sharing, adaptation, distribution and reproduction in any medium or format, as long as you give appropriate credit to the original author(s) and the source, provide a link to the Creative Commons licence, and indicate if changes were made. The images or other third party material in this article are included in the article's Creative Commons licence, unless indicated otherwise in a credit line to the material. If material is not included in the article's Creative Commons licence and your intended use is not permitted by statutory regulation or exceeds the permitted use, you will need to obtain permission directly from the copyright holder. To view a copy of this licence, visit http://creativecommons.org/licenses/by/4.0/.

\section{References}

1. de Ligt K, Luyendijk M, van Maaren M, de Munck L, Schreuder K, Siesling S et al (2018) Borstkanker in Nederland 26. https:// www.iknl.nl/kankersoorten/borstkanker/registratie/incidentie. Accessed 12 Mar 2021

2. Trialists TS, The UK (2008) Standardisation of breast radiotherapy (START) trial A of radiotherapy hypofractionation for treatment of early breast cancer: a randomised trial. Lancet Oncol 9(4):331-341

3. Agrawal RK, Aird EGA, Barrett JM, Barrett-Lee PJ, Bentzen SM, Bliss JM et al (2008) The UK standardisation of breast radiotherapy (START) trial B of radiotherapy hypofractionation for treatment of early breast cancer: a randomised trial. Lancet 371(9618):1098-1107

4. Correa C, Harris EE, Leonardi MC, Smith BD, Taghian AG, Thompson AM et al (2017) Accelerated partial breast irradiation: executive summary for the update of an astro evidence-based consensus statement. Pract Radiat Oncol 7(2):73-79

5. Bartelink H, Maingon P, Poortmans P, Weltens C, Fourquet A, Jager J et al (2015) Whole-breast irradiation with or without a boost for patients treated with breast-conserving surgery for early breast cancer: 20-year follow-up of a randomised phase 3 trial. Lancet Oncol 16(1):47-56

6. Marx RE, Ehler WJ, Tayapongsak P, Pierce LW (1990) Relationship of oxygen dose to angiogenesis induction in irradiated tissue. Am J Surg 160(5):519-524

7. Williamson RA (2007) An experimental study of the use of hyperbaric oxygen to reduce the side effects of radiation treatment for malignant disease. Int J Oral Maxillofac Surg 36(6):533-540
8. Tahir ARM, Westhuyzen J, Dass J, Collins MK, Webb R, Hewitt $S$ et al (2015) Hyperbaric oxygen therapy for chronic radiationinduced tissue injuries: Australasia's largest study. Asia Pac J Clin Oncol 11(1):68-77

9. Teguh DN, Levendag PC, Noever I, Voet P, van der Est H, van Rooij P et al (2009) Early hyperbaric oxygen therapy for reducing radiotherapy side effects: early results of a randomized trial in oropharyngeal and nasopharyngeal cancer. Int J Radiat Oncol Biol Phys 75(3):711-716

10. Hoggan BLCAL (2014) Systematic review of hyperbaric oxygen therapy for the treatment of non-neurological soft tissue radiationrelated injuries. Support Care Cancer 22:1715-1726

11. Teguh DN, Bol Raap R, Struikmans H, Verhoef C, Koppert LB, Koole A et al (2016) Hyperbaric oxygen therapy for late radiationinduced tissue toxicity: prospectively patient-reported outcome measures in breast cancer patients. Radiat Oncol 11(1):130

12. Carl UM, Feldmeier JJ, Schmitt G, Hartmann KA (2001) Hyperbaric oxygen therapy for late sequelae in women receiving radiation after breast-conserving surgery. Int J Radiat Oncol Biol Phys 49(4):1029-1031

13. Kot J, Desola J, Gata Simao A, Gough-Allen R, Houman R, Meliet J et al (2004) A European Code of Good Practice for HBO Therapy [Internet]. http://www.echm.org/documents/ECGP for HBO - May 2004.pdf. Accessed 3 May 2021

14. Aaronson NK, Ahmedzai S, Bergman B, Bullinger M, Cull A, Duez NJ et al (1993) The European organization for research and treatment of cancer QLQ-C30: A quality-of-life instrument for use in international clinical trials in oncology. J Natl Cancer Inst 85(5):365-376

15. Edmonds C (2016) Diving and subaquatic medicine, 5th edn. CRC Press, Boca Raton, pp 86-87

16. ONeill O, Brett K, Frank A (2020) Middle ear barotrauma. StatPearls, Treasure Island, FL (PMID: 29763026)

17. Claes J, Germonpre P, Van Rompaey V, Bourmanne E (2016) Ear, nose and throat and non-acoustic barotrauma. B-ENT 26(1):203-218

18. Giesinger JM, Loth FLC, Aaronson NK, Arraras JI, Caocci G, Efficace F et al (2020) Thresholds for clinical importance were established to improve interpretation of the EORTC QLQ-C30 in clinical practice and research. J Clin Epidemiol 118:1-8. https:// doi.org/10.1016/j.jclinepi.2019.10.003

19. Heyboer M, Sharma D, Santiago W, McCulloch N (2017) Hyperbaric oxygen therapy: side effects defined and quantified. Adv Wound Care 6(6):210-224

20. Clarke A, Clarke A, Brown R (1959) Regression to the mean-a confused concept. Brit J Psychol 51(2):105-117

21. Maraun MD, Gabriel S, Martin J (2011) The mythologization of regression towards the mean. Theory Psychol 21(6):762-784

22. Clinicaltrials.gov. Protocol HONEY study (2019) https://clini caltrials.gov/ct2/show/NCT04193722?term $=$ HONEY\&recrs $=$ $\mathrm{a} \&$ draw $=1 \&$ rank $=4$. Accessed 12 May 2021

Publisher's Note Springer Nature remains neutral with regard to jurisdictional claims in published maps and institutional affiliations. 